\title{
Analysis of the Integral Efficiency Indicator for Information Systems of the Cyclic Type Accounting for Weights
}

\author{
Tatyana Yuldasheva, Juliya Doronina \\ Departament of Automation and Computer Engineering, \\ Sevastopol National Technical University, Sevastopol, Ukraine \\ Email: yutatiys@mail.ru
}

Received March 6, 2013; revised April 12, 2013; accepted May 7, 2013

Copyright (C) 2013 Tatyana Yuldasheva, Juliya Doronina. This is an open access article distributed under the Creative Commons Attribution License, which permits unrestricted use, distribution, and reproduction in any medium, provided the original work is properly cited.

\begin{abstract}
The article is dedicated to the task of developing efficient means to analyze functioning of an information system of the cyclic type based on determining the integral performance criterion. We consider the subject area related to the analysis of moments of peak loads in teaching (admissions, conducting tests and examinations, accreditation of a university, etc.). The criterion is developed based on the analysis of all the processes occurring in the information system (IS) of a university and based on the selection of main factors affecting the change in the effective functioning of the university departments. Certain qualitative and quantitative parameters affecting the integral index of efficiency are considered at various times, since functioning of the whole system is cyclical. A weight for each criterion is accounted for in the integral indicator of efficiency. The proposed approach will allow for simplification of the research of efficiency characteristics of the information systems of the cyclic type based on the analysis of the integral coefficient.
\end{abstract}

Keywords: Information Systems of the Cyclic Type; Performance Indicator; Weights; Restructuring Databases

\section{Introduction}

Ensuring the effective functioning of an information system of the cyclic type in any subject area is important, for example, in ensuring continuous monitoring with cyclic periods of analysis of environmental parameters, environmental pollution, etc.

The university information system [1] is a complex, discrete, dynamic, and stochastic. An important issue in the development of any methodology is always selecting the primary set of indicators [2]. This is a serious problem because, on one hand, an excessive number of indicators can lead to a loss of simplicity and uniqueness in the interpretation of the result and its realization. On the other hand, reducing the number of indicators can lead to the loss of the integrated approach and undercount of some factors affecting the final evaluation of the system performance.

Specificity of the assessment tasks and forecasting of activities, such as personnel and financial information system of a university is that they are not formalized. The calculation of an efficiency indicator for the IS with the variable load criteria in personnel and financial databases includes a large number of different types of indicators, the impact of which on the whole system is different and depends on the type of the peak load.

\section{Data and Methodology}

The objective function of efficiency can be represented by the functional defined on the set of possible solutions, and during optimization it is necessary to find the solution that provides the maximum of this functional [3]. The main parameters that influence the system: $Q n-a$ load per unit of service personnel, Ksot-number of employees, Z - the department workload, Int-work rate, $\mathrm{Pr}$ - performance, $\mathrm{Tr}$ - the complexity, $\mathrm{Vt}$-volume of labor, $Q$ - quality of work processes, $C y$-vector of economic costs and others. All these parameters have different physical units. After the following normalization of these parameters:

$$
A_{i} \in\{Q n, K s o t, Z, I n t, \operatorname{Pr}, \operatorname{Tr}, V t \cdots\}
$$

by the variety of characteristic properties 


$$
b_{i}=\left\{b_{Q n}, b_{K s o t}, b_{z}, b_{\text {Int }}, b_{\mathrm{Pr}}, b_{T r}, b_{V t}\right\}
$$

we will obtain a set of normalized dimensionless parameters:

$$
K_{i}=A_{i} / b_{i} ; K_{i} \in\left\{K_{Q n}, K_{\text {Ksot }}, K_{Z}, K_{\text {Int }}, K_{\mathrm{Pr}}, K_{\text {Tr }}, K_{V t} \ldots\right\}(3)
$$

We divide the multitude (3) into the groups of normalized ratios of partial parameters by their functionality. For each group we will form the corresponding generalized normalized ratios.

In accordance with the main kinds of work that are performed in the appropriate departments we should identify the following generic coefficients: the effect of an employee, the effect of database (DB), the effect of documents, the effect of software and mathematical software, the effect for the student, the effect of organizational and legal support; the effect of the hardware and technical support.

To simplify the calculation of the integral efficiency ratio, the cyclic cyclical component is accounted for by the type of work, because in different periods of time, taking into account the type of load, preference is given to various indicators of system performance. We will consider the three main generalized indicator that have the greatest impact on the overall efficiency.

One of the important generalized coefficients is the indicator of employee efficiency $Y_{\text {sot }}$, which includes a group of normalized coefficients that describe the quality and quantity of work performed in the department.

An employee of the department performs the following types of work: making contracts with students, registration of contracts, sorting contracts, entering contracts into the database (DB), providing backing agreements to students, working with bank documents, working with the database, giving students copies of the contract, registration and issuance of certificates to students, processing applications for refund, working with students who are studying using budgetary loans, lease document preparation, working with the admissions committee, working with dean's and other university offices.

For determining the normalized ratio, the coefficient of the department load $Z$ was used [4]. Determination of the total amount of time used by the department to process the full day work load [5] (processing information flow) can be calculated by the formula:

$$
Z=\sum_{h=1}^{l} A_{h} \cdot Z_{h},
$$

where $A_{h}$ - The number of same type transactions carried out by the department during the day; $Z_{h}$-The necessary time required to conduct the $h$ type of work; l-The number of types of work carried out by the department.

The next important generalized coefficient is the index that shows performance of the database. There are various ways to reduce the time for querying a relational database. In [6] it is shown that one of these ways is to restructure the database based on the de-normalization. Under certain conditions it is possible to make such a decision, if at the same time the system performance increases. One of the most important normalized coefficients will be the time of the database query. The time of the query to the database:

$$
t=\sum_{j=1}^{n} t_{q_{j}} S_{q_{j}},
$$

where $q_{j}$-Text of the $j$ query; $t_{q_{j}}$-The time of the query $q_{j} ; s_{q_{j}}$-Number of executions for the $j$ query.

Time of execution of queries to the restructured database:

$$
t^{\prime}=\sum_{j=1}^{n} t_{q_{j}^{\prime}} s_{q_{j}^{\prime}}+\sum t_{m} s_{m},
$$

where $q_{j}^{\prime}$-The modified request to the restructured database; $t_{m}$-Time to perform additional processing to maintain the integrity of data during modification of relations; $s_{m}-$ Number of runs of the additional code for the relevant time.

Statistical studies show that, after the database demoralization the ratio is $t^{\prime} / t \square 1$.

The main indicators of reliability of software characterize the presence of program errors (manufacturing defects), but none of them characterize the nature of these errors and their possible consequences [7]. It is therefore proposed to accept the ratio of average error severity as a basic ratio or reliability:

$$
B=1 / Q \times \sum_{j=1}^{n}\left(b_{j} \cdot p_{j} \cdot r_{j}\right)
$$

where $Q-$ The probability of software failure during the denormalize of the database;

$b_{j}$-Membership function of the severity of the error during denormalization of the database occurred during the input $\mathrm{j}$ of data to most severe consequences;

$p_{j}$ - The probability of entering the $j$ input data set during denormalization of the database;

$r_{j}$-Dichotomous variable equal to 1 if during the $j$ input of data there was a failure, and otherwise equal to 0 ;

$n$-The total number of queries.

The value of the reliability of average error severity lies in the interval $[0,1]$. The closer it to 1 , the harder the consequences of the software errors and the less reliable the software is. The proximity of average error severity to zero shows minor effects of software errors.

For the generalized use of the coefficients (4)-(7) it is necessary to determine the weight of the corresponding factor to the current coefficient, reflecting the degree of 
its importance among all the selected factors. On the basis of expert opinions, scores are provided for each factor and the total weighted score is calculated according to the rules of linear convolution. This weighed estimate is the characteristic of the integral coefficient of efficiency. The expression for $E$ is as follows:

$$
E=\sum_{i=1}^{m} W_{i} \cdot\left(\Delta t_{i} \cdot B_{i} \cdot 1 / Z_{i}\right) / \sum_{i=1}^{m} W_{i},
$$

where $E$ - The integral index of the information system efficiency;

$\Delta t_{i}$ - An indicator of difference in the time of query executing to the original and restructured database;

$W_{i}$ - Weight (degree of importance) of the factor $i$;

$B_{i}$ - An indicator of the reliability of the software;

$Z_{i}$ - The value of the total amount of time for the department to complete all the daily work load;

$m$-Number of factors;

$i$-Factor index.

We divide the sum of weight coefficients $\sum_{i=1}^{m} W_{i}$ into the subsets of generalized coefficients accounting for their weights depending on the type of load at different periods of the system cyclces: $\sum_{j=1}^{n} W_{j}^{\Delta t}, \sum_{j=1}^{n} W_{j}^{B}$, $\sum_{h=1}^{l} W_{h}^{Z}$. Accounting for (4)-(7), the expression (8) is the following:

$$
E=\frac{\sum_{j=1}^{n} W_{j}^{\Delta t} \times\left[\sum_{j=1}^{n} t_{q_{j}} s_{q_{j}}-\sum_{j=1}^{n} t_{q_{j}^{\prime}} S_{q_{j}^{\prime}}-\sum_{j=1}^{n} t_{m} s_{m}\right]}{\sum_{j=1}^{n} W_{j}^{b} \times Q \sum_{j=1}^{n}\left(b_{j} p_{j} r_{j}\right) \sum_{h=1}^{l} W_{h}^{z} \times \sum_{h=1}^{l} A_{h} Z_{h}}
$$

where $\sum_{j=1}^{n} W_{j}^{\Delta t}$-Weight (degree of importance) of the factor $j$, that shows performance of the database;

$q_{j}$-Text of the $j$ query;

$t_{q_{j}}$ - The time of the query $q_{j}$;

$s_{q_{j}}$-Number of executions for the $j$ query;

$q_{j}^{\prime}$-The modified request to the restructured database;

$t_{m}$-Time to perform additional processing to maintain the integrity of data during modification of relations;

$s_{m}$-Number of runs of the additional code for the relevant time;

$\sum_{h=1}^{l} W_{h}^{Z}$-Weight (degree of importance) of the factor

$h$, that shows performance of the department load;

$Q$ - The probability of software failure during the denormalize of the database;

$b_{j}$-Membership function of the severity of the error during denormalization of the database occurred during the input $j$ of data to most severe consequences;

$P_{i}$ - The probability of entering the $j$ input data set during denormalization of the database;

$r_{j}$-Dichotomous variable equal to 1 if during the $j$ input of data there was a failure, and otherwise equal to 0 ;

$n$-The total number of queries;

$\sum_{j=1}^{n} W_{j}^{B}$-Weight (degree of importance) of the factor $j$, that shows average error severity of software;

$A_{h}$ - the number of same type transactions carried out by the department during the day;

$Z_{h}$ - The necessary time required to conduct the $h$ type of work;

l-The number of types of work carried out by the department.

Weight corresponding to the factors is normalized. Thus, the weight of each of the generalized coefficient gives an estimate of the effectiveness of the information system of the cyclic type depending on the priority of work performed. Statistical analysis shows that the effecttive use of such information system mostly depends on the time of query execution to the database. The results are presented in Figure 1.

\section{Research Results}

The paper presents the main aspects of the efficiency evaluation for the information system of the cyclic type (hybrid model) by the example of a university personnel finance information system. The main indicators to be measured and the factors of the cycle, such as peak loads, are considered when priorities change for each indicator during all system efficiency assessment. A set of indicators that describing the activities of the information system of a university, is selected in such a way that all processes affecting the final performance are covered most fully.

The research results have shown that to increase the

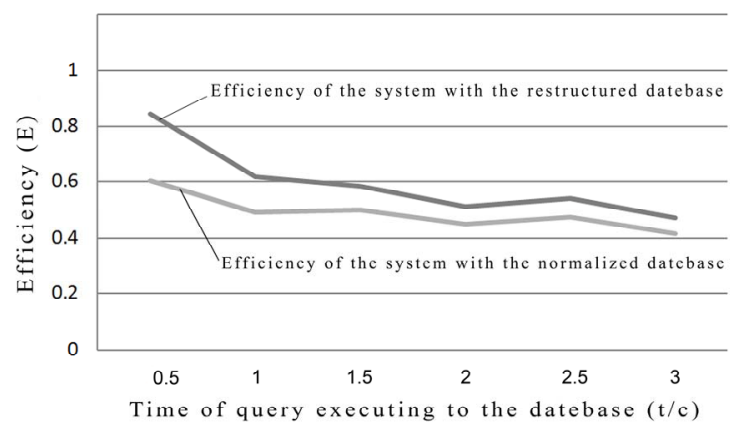

Figure 1. Graphs of efficiency changes for the information system (E) depending on the time difference of the query execution (t) between the normalized and restructured database. 
efficiency of the personnel finance information system it is necessary to vary the degree of factor importance in the process of system functioning that is fine tuning during when revealing the degree of influence for each facto. For example, the greatest impact on the efficiency ratio of the university personnel finance information system has the time of query execution to the database but, in the same time, the probability of the system failure increases due to the violation of integrity. Subtle analysis of these and other similar situations will clarify the integral indicator of efficiency of the information system of the cyclic type, which, in turn, will increase the quality of the evaluation for the systems of such class.

At present, this method of determination of the integral criterion of efficiency is applied in personnel and financial information system of the cyclic type of Sevastopol National Technical University. Account of the importance of different factors in the process of functioning system has allowed to increase the efficiency of the information system by $14 \%$. Further studies are being conducted in this area and the method is being approbated at the moment.

\section{REFERENCES}

[1] A. D. Bolgarov, N. N. Babak, O. K. Pogydin and C. A.
Koba, "Method of Formation Integral Indicator Effectiveness of Projects' Informatization," Vol. 29, No. 3, 2011, pp. $95-102$.

[2] B. V. Semkin, O. N. Pyatkovskiy and N. P. Scherbakov, "Evaluation of Workload Educational Complexes," 2011. http://edu.secna.ru/media/f/razrabotka.pdf

[3] O. S. Litvinskaya and I. I. Salnikov, "Application of the Target Function in the Decision-Making Process Using the Choice of a Variant of Realization of the Algorithm for Sequential Data Processing," No. 3, 2009, pp. 115125.

https://e.mail.ru/cgi-bin/ajax_attach_action?id=13547294 $130000000617 \&$ \&av $=0$

[4] Yu. V. Doronina, "Reengineering of Monitoring Information Systems of Cyclic Types," East-European Journal of Advanced Technologies, Vol. 55, No. 1-2, 2012, pp. 1214.

[5] I. Yankovskiy, "Modeling of the Workload of the Staff of the Institution," 2003.

http://www.nbrb.by/bv/narch/234/16.pdf

[6] A. B. Kyngyrcev and S. L. Zinovatnaya, "An Analysis of the Restructuring of the Database by the Method of Introduction of Descending Demoralization," Written Proceedings of Odessa Polytechnic University, Vol. 25, No. 1, 2006. pp. 104-109.

[7] O. O. Pavlovskaya, "Static Methods of Assessment of Software," Computer Technologies, Management, Radio Electronics, Vol. 26, No. 10, 2007, pp. 35-37. 